\title{
Conductance through a single atom
}

\author{
H. Mehrez and S. Ciraci \\ Department of Physics, Bilkent University, Bilkent 06533, Ankara, Turkey
}

A. Buldum ${ }^{*}$ and Inder P. Batra

IBM Almaden Research Center, San Jose, California 95120-6099

(Received 29 October 1996)

\begin{abstract}
In this paper we present an analysis of conduction through a single atom between two metal electrodes. Based on $a b$ initio total-energy and electronic-structure calculations, and molecular-dynamics simulations using the embedded-atom model, we show that the conductance through an atom depends on the electronic structure of both the single atom and the metal electrodes, as well as the binding structure between the single atom and the surfaces of the metal electrodes. Our results enable us to interpret experimental results obtained by using a mechanical break junction on atomic-scale wires. [S0163-1829(97)50904-0]
\end{abstract}

How the conductance of a mesoscopic object depends on its size and dimensionality has always been an attractive as well as intriguing subject for researchers. As early as 1957, Landauer ${ }^{1}$ proposed that the conduction in a solid is a scattering event, and that transport is the consequence of the incident current flux. Based on the counting arguments of the transmissions $T$, and reflections $R$, he derived his famous formula for the conductance $G=\left(2 e^{2} / h\right)(T / R)$. Almost three decades ago Sharvin ${ }^{2}$ pointed out the resistance of a ballistic channel (or point contact) and developed a formalism in the semiclassical regime. Nowadays, almost defectfree electronic devices have been fabricated, which have dimensions, in one or more directions, on the quantum scale. The two-terminal conductance of such a quasi-onedimensional device (or constriction) fabricated from the high mobility GaAs- $\mathrm{Al}_{1-x} \mathrm{Ga}_{x} \mathrm{As}$ heterojunction ${ }^{3,4}$ (which has width $w$ in the range of Fermi wavelength $\lambda_{F}$, and length $l$ smaller than the electron mean free path) were found to change with $w$ approximately in steps of $2 e^{2} / h$. This observation has been interpreted as the quantization of ballistic conductance. It becomes clear from subsequent studies ${ }^{5-7}$ that the motion of electron in the constriction is transversally quantized if $l \sim \lambda_{F}$, and then its level spacings become in the range of a few Kelvin, if $w$ is in the range of $\lambda_{F}$. An $n$-fold degenerate current-carrying state becomes conducting and hence $G$ increases by $2 e^{2} n / h$ whenever its energy coincides with $E_{F}$; this situation is denoted as the opening of an $n$-fold channel. The variation of $G$ with $w$ displays sharp steps of integer multiples of $2 e^{2} / h$, and flat plateaus form between two consecutive steps provided that the potential in the constriction is uniform, temperature and bias voltage are low, and tunneling contribution is negligible. Otherwise, conduction channels mix significantly, the opening of channels are delayed, sharp step structure is smeared out and step heights are lowered. ${ }^{7}$

The atomic-size point contact was produced first by the scanning tunneling microscope (STM): By displacing the tip towards the sample surface, the conductance were measured in a wide range covering tunneling and ballistic regimes. ${ }^{8}$ The experimental $\log G$ versus tip displacement either exhibits a linear variation and then saturation at the first plateau before a jump, or it ends the linear variation directly by a jump. The jumps occur due to the sudden enlargement of the contact by the rearrangement of atoms at the contact. ${ }^{9}$ Furthermore, recent work on atomic-scale wires obtained by mechanical break junction ${ }^{10}$ showed that the conductance versus stretch, $s$ curve, $G(s)$ passes through a plateau just before the break with $G \sim 2 e^{2} / h$ (for Al wire) or $G \sim 4 e^{2} / h$ (for Pt wire). While $G$ increases slightly with $s$ for $\mathrm{Al}$ and $\mathrm{Pt}$ wires, it becomes rather flat for the $\mathrm{Cu}$ wire. The above behaviors of $G(s)$ are related with the point contact formed by an atom either at the apex of the tip or between two metal electrodes of the break junction, and point to the conductance through a single atom. Different aspects of this issue were considered in a number of previous studies. ${ }^{7,9-13}$ In this work, we show that the site and structure of binding are crucial for the conductance through a single atom between two metal electrodes.

We performed $a b$ initio self-consistent-field (SCF) pseudopotential calculations within the local density approximation using Wigner exchange-correlation potential. The junction consisting of an atom which is attached to the surfaces of two metal electrodes is represented by two $\mathrm{Al}(001)$ slabs connected by a single $\mathrm{Al}$ atom. This structure is treated by a repeating cell that contains $55 \mathrm{Al}$ atoms. The wave function is expressed by $\sim 2500$ plane waves, which corresponds to the kinetic energy cutoff $|\vec{k}+\vec{G}|^{2} \leqslant 6 \mathrm{Ry}$. At equilibrium (or at zero tensile stress) condition the lowest total energy $E_{T}$ is attained when a single $\mathrm{Al}$ is attached to the (hallow) $H$ sites of both slab surfaces with the bond distance of $d \sim 2.1 \AA$. This corresponds to the separation of two $\operatorname{Al}(001)$ slabs $\xi=\xi_{e}=2 d$. As $\xi$ is increasing (or electrodes are pulled apart), the binding energy (and hence the magnitude of $E_{T}$ ) is lowered, and eventually the (top) $T$ site, which is only a local minimum in the Born-Oppenheimer surface, becomes favored. ${ }^{14}$ When the separation between electrodes reaches a critical value $\left(\xi=\xi_{c}\right)$, single atom stays attached (absorbed) only to one of the electrode surfaces, so the junction is broken. In a separate study, we also performed the simulation of $\mathrm{Cu}$ nanowires under tensile stress by using the molecular dynamics method with embedded atom 
potential, ${ }^{15}$ and examined the modifications of atomic structures. In the course of pulling, the nanowire elongates in consecutive quasielastic (continuous) and yielding (discontinuous) stages. In the yielding stage, a new layer with a relatively smaller cross section is formed and hence the number of atoms at the neck is reduced discontinuously when the stretch $s$ attains certain values. Concomitantly, the tensile force decreases suddenly. While the atoms in the neck region keep the $H$ site registry during the quasielastic stage, short atomic chain(s) or a single atom neck forms shortly before the break as a result of transition from the $H$-site registry to the $T$-site registry. ${ }^{16}$ As we show later in this paper this transition has important implications. The metal electrodes have quasicontinuous electronic energy states $\epsilon_{k}$, which are filled up to $E_{F}$, whereas the single atom at the neck has discrete energy states $\epsilon_{n}$. Some of the $\epsilon_{n}$ 's are occupied, the rest lie above $E_{F}$. Current-carrying states can form between two electrodes (or reservoirs), and open a channel if the incoming electron from one electrode (with finite longitudinal wave vector $k_{z}$, and energy $\epsilon_{k} \sim E_{F}$ can be matched to an empty outgoing state of the other electrode through the discrete states of a single atom, ${ }^{13} \epsilon_{n} \sim E_{F}$. If $\epsilon_{k}<\epsilon_{n}, \epsilon_{n}$ sets an effective barrier for transport, $\phi_{\text {eff }}=\epsilon_{n}-\epsilon_{k}$, so that the incoming electron can tunnel only. The transition from the tunneling to the ballistic transport occurs, however, when $\epsilon_{n}$ becomes aligned with $E_{F}$ and hence $\phi_{\text {eff }}$ collapses as a result of deformation. The saturation of $G(s)$ at the first plateau in Ref. 8 was explained by this process, which took place before the first mechanical instability. ${ }^{9}$ Here $G$ may be smaller than $2 e^{2} / h$ because of saddle point effect and backscattering. An alternative understanding of the transport through the above neck can be provided by the theory of Kalmeyer and Laughlin: ${ }^{11,17,18}$ According to the Newns and Anderson theory, ${ }^{19}$ the state $\epsilon_{n}$ of the single atom that is absorbed on the metal surface is broadened and becomes a resonance with a Lorentzian distribution $\rho_{a}(\epsilon)=(\Gamma /$ $\pi)\left[\left(\epsilon-\epsilon_{n}-\Lambda\right)^{2}-\Gamma^{2}\right]^{-1}$. It is centered at $\epsilon=\epsilon_{n}+\Lambda$ with full width at half maximum $\Gamma$. If $\epsilon_{k}$ of the incoming state is aligned with the center of resonance, the conductance can attain the quantum value $2 e^{2} / h$ (excluding effects that delay the channel opening). Otherwise (i.e., if $\epsilon_{n}+\Gamma>\epsilon_{k}$ ) $G$ is reduced by $\rho_{a}\left(\epsilon_{k}\right) / \rho_{a}\left[\epsilon_{n}+\Lambda\right]$. It is clear from earlier works ${ }^{9,10,13}$ and from this discussion that the conductance $G$ through the single atom depends on the electronic structure of electrodes, as well as discrete energy states of the neck atom connecting two electrodes.

We now investigate the effect of the binding structure of the single atom with the metal electrodes. To understand this phenomenon, we first calculate the electronic energy structure of an infinite chain of $\mathrm{Al}$ atoms with optimized interatomic distance $a$. The band structure is illustrated in Fig. 1. The lowest band (or $\sigma$ band) is made from the combination of $s+p_{z}$ orbitals located below $E_{F}$. Normally, this band does not contribute to the transport. The second band (or $\pi$ band) is a doubly degenerate band; it originates from the linear combination of $3 p_{x}$ and $3 p_{y}$ orbitals of $\mathrm{Al}$ atoms and crosses the Fermi level at $\pi / 4 a$. The $\mathrm{Al}$ chain undergoes a small Pierels distortion, and its ballistic conductance $G=4 e^{2} / h$ when the chain is perfect. On the other hand, the conductance of infinite and perfect chain of $\mathrm{Na}$ is only $2 e^{2} / h$ (half the conductance of Al chain), prior to the Pierels

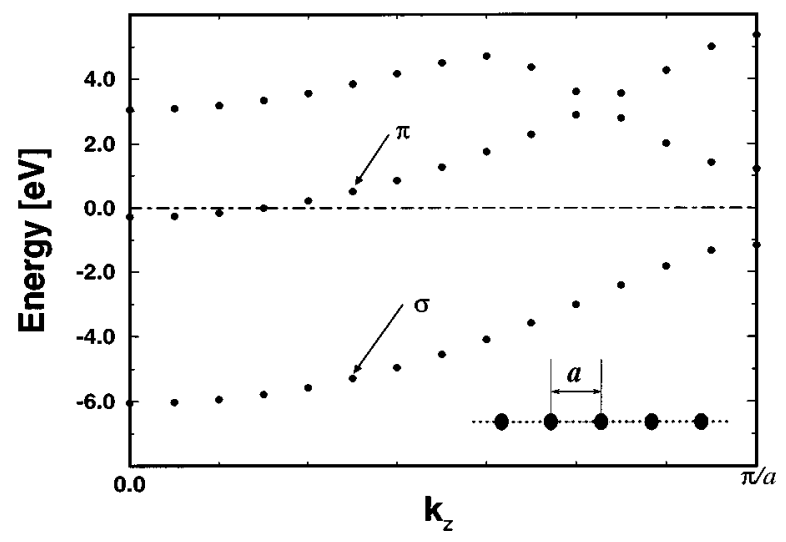

FIG. 1. Energy band structure of the infinite Al chain calculated by the SCF pseudopotential method with the local density approximation. The lattice parameter $a$ is optimized at $2.4 \AA$. The $\sigma$ and doubly degenerate $\pi$ bands are shown. The zero of energy is set to $E_{F}$ shown by dash-dotted line.

distortion. This shows that the value of $G$ is determined by the bond formation, as well as by the valence electronic structure of constituent atoms.

Having discussed the conductance of perfect metal chains, we consider now the junction of a single $\mathrm{Al}$ atom between two $\mathrm{Al}(001)$ slabs which is relevant for the experiment. ${ }^{10}$ The conductance through a single $\mathrm{Al}$ atom was calculated earlier by using different methods. The theoretical value calculated for the $\mathrm{Al}$ atom adsorbed between two jellium electrodes at equilibrium distance, ${ }^{11}$ and that calculated for the contact of the $\mathrm{Al}$ tip on the $\mathrm{Al}(111)$ surface by using a potential that is parameterized from SCF results ${ }^{13}$ came out larger than $2 e^{2} / h$. The corresponding value measured by the mechanical break junction ${ }^{10}$ is in the range of $2 e^{2} / h$ just before the break. The discrepancy between the theoretical and experimental values and also the observed positive slope $^{10}$ of $G(s)$ curve (i.e., $G$ increasing with the stretch) are important issues. These issues, as well as the question of whether the conductance through a single atom depends on the binding configuration will be resolved in the following analysis. To this end, we calculate the local density of states of the junction at the single Al, $\rho_{a}(\epsilon)$ for different binding structure of $\mathrm{Al}$ atom (i.e., different $\xi$ and different binding states).

Our results for $\rho_{a}(\epsilon)$ obtained from SCF pseudopotential calculations described at the beginning are illustrated in Fig. 2. The junction consisting of $55 \mathrm{Al}$ atoms is schematically described by the inset. For the H-site binding, $\rho_{a}(\epsilon)$ decreases with the stretch $s$. For example, when the distance between the single $\mathrm{Al}$ and slab surface $d=\xi / 2$ is stretched by $30 \%$ the local density of states of the single $\mathrm{Al}$ atom at $E_{F}, \rho_{a}\left(E_{F}\right)$ is reduced by $42 \%$. According to the theory of Kalmeyer and Laughlin, ${ }^{17}$ this implies a significant decrease of conductance upon stretch. Note that the neck is reduced from a few atoms to a single atom after the junction undergoes the last yielding stage. ${ }^{10}$ According to the results of molecular dynamics simulations, the neck is already strongly stretched under a significant tensile stress in the last elastic stage before the break. Consequently, the distance of the single $\mathrm{Al}$ atom from the surfaces of electrodes $d$ is larger 


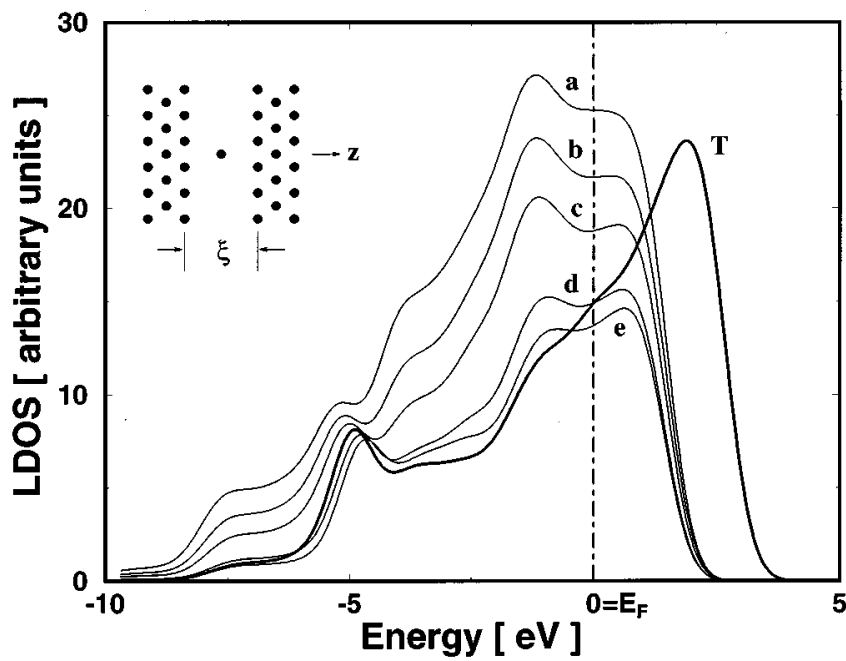

FIG. 2. The local density of states $\left[\operatorname{LDOS}\right.$ or $\left.\rho_{a}(E)\right]$ at a single $\mathrm{Al}$ atom. The junction shown by the inset consists of an $\mathrm{Al}$ atom adsorbed to the $\mathrm{Al}(001)$ slabs. The $H$-site configurations $a, b, c, d$, and $e$ correspond to $d=\xi / 2=2.1 \AA$ (equilibrium distance), $2.25 \AA$, $2.38 \AA$, $2.65 \AA$, and $2.75 \AA$, respectively. The curve $T$ is the LDOS of Al bound to the $T$ site with $d=2.75 \AA$.

than the equilibrium value. Electronic structure calculations of the junction for the $T$-site and stretched $H$-site registry for $d=2.75 \AA$ show dramatic changes relative to the infinite chain. The energy levels of the junction shift also by stretch, and/or by transforming from one registry to another. For example, the doubly degenerate $\pi$ band of the infinite chain, which is responsible from the conductance of $4 e^{2} / h$, becomes a doubly degenerate state in the junction below the Fermi level. In the stretched $H$-site registry, this state is relatively closer to $E_{F}$, but its charge is transferred to the metal surfaces. This explains how the high $\rho_{a}\left(E_{F}\right)$ on the single Al bound to the $H$ sites of the $\mathrm{Al}(001)$ surfaces decreases with the stretch, whereas the $\sigma$ band of the infinite $\mathrm{Al}$ chain that is totally below $E_{F}$ changes into a singlet slightly above $E_{F}$ in the junction. This state becomes responsible for the conduction. In the $T$-site registry, this state is closer to $E_{F}$ than that in the $H$-site registry. In Fig. 3, we show the calculated charge density contour plots of the $\sigma$ and $\pi$ states at the $T$-site registry.

In view of the above discussion and results illustrated in Fig. 2, the neck atom with $H$-site registry may have reduced $G$ in the mechanical break junction since its $\rho_{a}\left(E_{F}\right)$ is decreased as a result of stretch. Since $\rho_{a}\left(E_{F}\right)$ for $d=2.75 \AA$ is larger at the $T$-site registry than at the $H$-site registry, we expect a slight increase of $G$ as a result of this structural transition induced by the tensile stress. This provides an explanation as to why $G(s)$ of the Al neck increases with stretch at the last plateau.

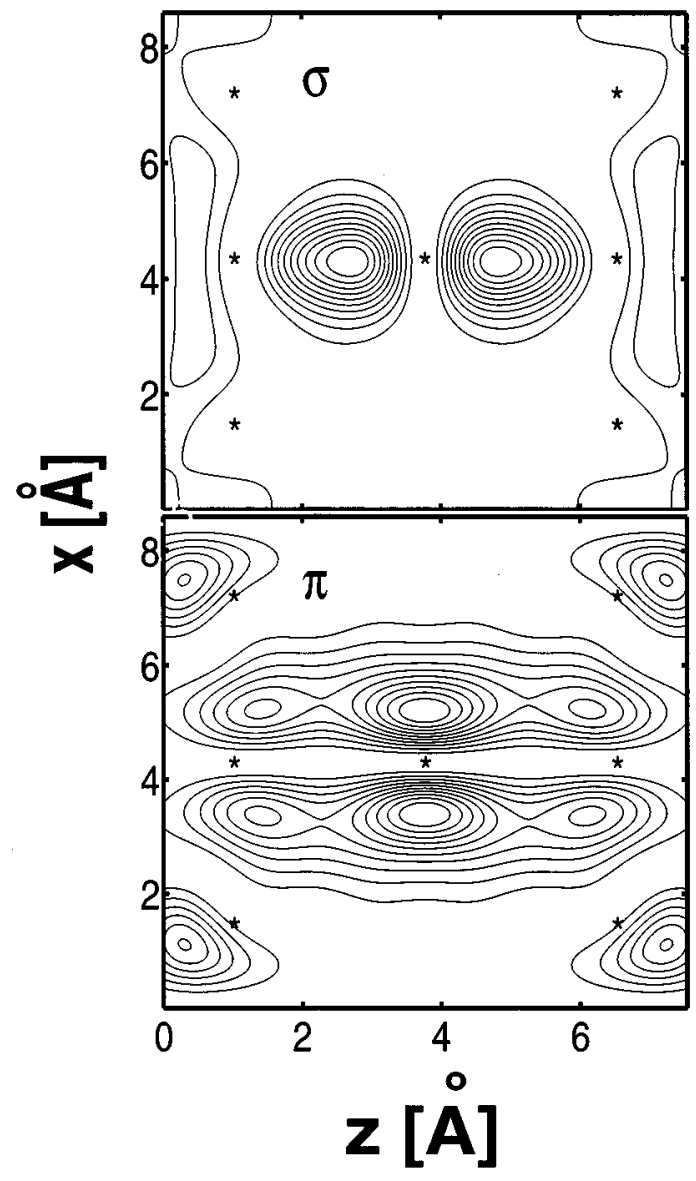

FIG. 3. The charge density contour plots of the $\sigma$ and $\pi$ states of the junction, which consists of a single $\mathrm{Al}$ atom bound to two $\mathrm{Al}(001)$ surfaces at the $T$ site with $d=2.75 \AA$. The positions of $\mathrm{Al}$ atoms are indicated by stars. The maximum charge density and contour spacing for the $\sigma$ state $(\pi$ state $)$ are 1.15 $\times 10^{-2}$ electrons $/ \AA^{3}$ and $1.03 \times 10^{-3}$ electrons $/ \AA^{3}\left(4.15 \times 10^{-3}\right.$ electrons $/ \AA^{3}$ and $3.8 \times 10^{-4}$ electrons $/ \AA^{3}$ ), respectively.

In conclusion, we showed that the conductance through a single atom neck formed between two metal surfaces depends on the electronic structure of metal electrodes and single atom as well as the detailed binding structure with electrodes.

H.M. and S.C. thank the Computer Center of Middle East Technical University for the time provided in the parallel computer facility. A.B. thanks TÜBITAK and IBM Corporation for their support, providing his visit to IBM-SP Briefing Center at Almaden Research Laboratory in San Jose, California.
*Permanent address: Department of Physics, Bilkent University, Bilkent 06533, Ankara Turkey.

${ }^{1}$ R. Landauer, IBM J. Res. Dev. 1, 223 (1957); Philos. Mag. 21, 863 (1970).

${ }^{2}$ Yu. V. Sharvin, Zh. Eksp. Teor. Fiz. 48, 984 (1965) [Sov. Phys. JETP 21, 655 (1965)].
${ }^{3}$ B. J. van Wees, H. van Houten, C. W. J. Beenakker, J. G. Williamson, L. P. Kouwenhoven, D. van der Marel, and C. T. Foxon Phys. Rev. Lett. 60, 848 (1988).

${ }^{4}$ D. A. Wharam, T. J. Thorton, R. Newbury, M. Pepper, H. Ahmet, J. E. F. Frost, D. G. Peacock, D. A. Ritchie, and G.A.C Jones, J. Phys. C 21, L209 (1988). 
${ }^{5}$ M. Büttiker, Y. Imry, R. Landauer, and S. Pinhas, Phys. Rev. B 31, 6207 (1985); see also Y. Imry, Directions in Condensed Matter Physics, edited by G. Grinstein and G. Mazenko (World Scientific, Singapore, 1986), p. 101.

${ }^{6}$ C. W. J. Beenakker and H. van Houten, in Solid State Physics, edited by H. Ehrenreich and D. Turnbull (Academic, New York, 1991), Vol. 44, p. 1.

${ }^{7}$ Extensive analysis and several relevant references can be found in E. Tekman and S. Ciraci, Phys. Rev. B 43, 7145 (1991); see also ibid. 39, 8772 (1989).

${ }^{8}$ J. K. Gimzewski and R. Möller, Phys. Rev. B 36, 1284 (1987).

${ }^{9}$ S. Ciraci and E. Tekman, Phys. Rev. B 40, 11969 (1989).

${ }^{10}$ J. M. Krans, C. J. Müller, I. K. Yanson, Th. C. M. Govaert, R. Hesper, and J. M. van Ruitenbeek, Phys. Rev. B 48, 14721 (1993).

${ }^{11}$ N. D. Lang, Phys. Rev. B 36, 8173 (1987); ibid. 52, 5335 (1995); A. Yazdani, D. M. Eigler, and N. D. Lang, Science 272, 1921 (1995).

${ }^{12}$ C. Joachim, J. K. Gimzewski, R. R. Schlittler, and C. Chavy, Phys. Rev. Lett. 74, 2102 (1995).

${ }^{13}$ E. Tekman and S. Ciraci, Phys. Rev. B 42, 1860 (1990); S. Ciraci, A. Baratoff, and I. P. Batra, ibid. 42, 7618 (1990). In these works, the interatomic separations were taken fixed at their bulk equilibrium values and the conductance was calculated in a wide range of separation while the tip is approaching the surface including also the tunneling before the point contact. The hysteric mechanical instability or jump to contact were not taken into account. In the present work, the last step of pulling where the neck is formed after the nanoindentation is investigated. It becomes clear that in the last step of pulling, the discontinuous atomic rearrangement as well as the elastic deformations are crucial for the electron transport through atomic-size neck.

${ }^{14}$ S. Ciraci, E. Tekman, A. Baratoff, and I. P. Batra, Phys. Rev. B 46, 10411 (1992).

${ }^{15}$ M. S. Daw and M. I. Baskes, Phys. Rev. B 29, 6443 (1984); S. M. Foiles and M. S. Daw, ibid. 33, 7983 (1986); M. S. Daw, ibid. 39, 7441 (1989).

${ }^{16}$ The $T$-site registry denotes the configuration in which atoms form a chain around the $T$ sites. Note that for some systems the break can occur before the formation of chain structure.

${ }^{17}$ V. Kalmeyer and R. B. Laughlin, Phys. Rev. B 35, 9805 (1987).

${ }^{18}$ A. Buldum and S. Ciraci, Phys. Rev. B 54, 2175 (1996).

${ }^{19}$ P. W. Anderson, Phys. Rev. 124, 41 (1961); D. M. Newns, ibid. 178, 123 (1969). 